\title{
CHANCE CONSTRAINT OPTIMIZATION OF A COMPLEX SYSTEM: APPLICATION TO THE FATIGUE DESIGN OF A FLOATING OFFSHORE WIND TURBINE MOORING SYSTEM
}

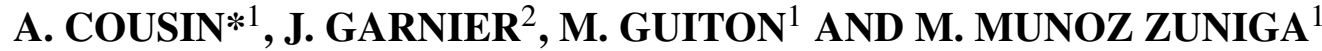 \\ ${ }^{1}$ IFP Energies Nouvelles \\ 1-4 Avenue du Bois Préau, 92852 Rueil-Malmaison \\ alexis.cousin@ifpen.fr, martin.guiton@ifpen.fr, miguel.munoz-zuniga@ifpen.fr \\ ${ }^{2}$ CMAP, Ecole Polytechnique \\ Route de Saclay, 91120 Palaiseau \\ josselin.garnier@polytechnique.edu
}

Key words: RBDO, Extreme Value Theory, Adaptive Kriging, Monte Carlo, Floating Offshore Wind Turbine, Fatigue

\begin{abstract}
In this paper, we seek to minimize the cost of the anchoring system of a floating offshore wind turbine under reliability constraints. Taking into account the uncertainties on the model, on the resistance threshold and on the environmental conditions implies constraints expressed as probabilities depending on random vectors and a piecewise stationary Gaussian process. The main difficulty of the studied problem is to compute these probabilities since reliability methods require many calls to the simulator of the system. We propose in this paper a two-step methodology allowing to solve the optimization problem with a reasonable number of calls to the simulator. First, we exploit the properties of the problem to reformulate the constraints into easier to compute ones. Then we propose a new approach based on adaptive kriging well suited to the reformulated problem: AK-ECO.
\end{abstract}

\section{INTRODUCTION}

Floating offshore wind turbines (FOWT) enable to install high power turbines with favorable wind conditions. Their economic feasibility requires an optimization of the additional cost due to the floater equipment. The goal of this paper is to propose an innovative cost-efficient optimization approach for configurating the mooring system of a modified version of the NREL 5MW-DeepCWind FOWT [1]. The mooring system must limit the floater movements to ensure the turbine production, avoid compression in the mooring lines and withstand the damage caused by fatigue. The resulting constraints inherit the randomness of the marine conditions, of the material properties and model parameters. Therefore, we face an optimization problem with a deterministic cost function and probabilistic constraints with very high confidence levels $\left(10^{-4}\right.$ for annual failure probability in [2]). A naive approach such as the Monte Carlo method is not feasible due to the time required in FOWT simulations since several thousand simulations are required to estimate a deterministic fatigue life [3].

To overcome this difficulty, we propose a methodology that takes into account the nature of the con- 
straints to solve the problem within a reasonable computational time. First, we use the Extreme Value Theory to reformulate the surge and tension constraints. The computations are performed in the frequency domain [4] and the fatigue damage is estimated with the spectral Dirlik empirical formulae [5]. In order to determine the feasibility region defined by the probabilistic constraints, metamodels are then built and sequentially enriched according to a new procedure introduced in this paper.

We emphasize that the proposed methodology is based on two important simplifications. First, in order to work in the frequency domain, the movements of the structure are solution of a linearized equation of movement and only the first order hydrodynamic loads are taken into account. Moreover, even though the wind speed variations may contribute to the mooring line fatigue, we consider here only constant wind forces on the structure for simplicity.

\section{PROBLEM FORMULATION}

We seek to minimize the cost of the mooring system of a FOWT while respecting reliability constraints. We thus face an optimization problem whose characteristics are presented in this section.

\subsection{Design variables and design space}

The cost of the mooring system and the constraints depend on three design variables: the change $d_{1}$ in length of the mooring line from the nominal value of $841.2 \mathrm{~m}$ (its domain is $[-2,2]$ (in $\mathrm{m}$ )), the lineic mass $d_{2} \in[70,180]$ (in $\mathrm{kg} / \mathrm{m}$ ), the position $d_{3}$ of the connection of the lines to the columns of the floater (taking values between 0 and 1 which respectively correspond to the connection at the bottom and at the top of the columns). We denote $d=\left(d_{1}, d_{2}, d_{3}\right)$ and the design space $\Omega_{d}=[-2,2] \times[70,180] \times[0,1]$.
2.2 Envirommental condlitions
The movements of the structure are determined from the environmental loads occurring during the con-
sidered period $[0, T]$ ( $T$ is one year $)$ and in particular wave loading. For a particular sea state defined below, which lasts for three hours, the sea elevation is modeled by a stationary stochastic Gaussian pro-

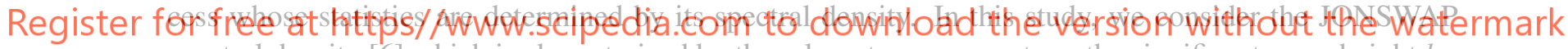
spectral density [6] which is characterized by three long term parameters: the significant wave height $h_{s}$, the peak period $t_{p}$ and the mean wind speed $u$.

To account for the wave distribution, the period $[0, T]$ is decomposed into $n_{T}$ intervals $I_{i}=[(i-1) \Delta T, i \Delta T]$, $\left(i=1, \ldots, n_{T}\right)$ where $\Delta T$ is three hours. At each interval $I_{i},\left(h_{s}, t_{p}, u\right)_{i}$ define a sea state with a process of sea elevation. Moreover they are the realizations of the random vector $\left(H_{s}, T_{p}, U\right)_{i}$ with known joint law. In this work, for simplicity and without loss of generality, we consider only seven possible outcomes of $\left(H_{s}, T_{p}, U\right)_{i}$ denoted $\left(h_{s}^{j}, t_{p}^{j}, u^{j}\right),(j=1, \ldots, 7)$ with probability $p^{j}$ (with $\left.\sum_{j=1}^{7} p^{j}=1\right)$. Those outcomes were selected to represent the long term parameters joint distribution as best as possible. Finally, the random vectors $\left(H_{s}, T_{p}, U\right)_{i},\left(i=1, \ldots, n_{T}\right)$ are i.i.d. We denote $\eta_{\left(h_{s}, t_{p}, u\right)_{i}}$ the stationary process on the $i$-th interval, $X_{L T}$ the sequence of random triplets $\left\{\left(H_{s}, T_{p}, U\right)_{i}, i=1, \ldots, n_{T}\right\}$ and $\eta_{x_{L T}}$ the piecewise stationary process equal to $\eta_{\left(h_{s}, t_{p}, u\right)_{i}}$ on each interval $I_{i}$.

\subsection{Surge, tension and fatigue}

At the end of the optimization problem, the chosen design variables must restrict the platform movements: the horizontal shifting of the structure (called surge) must be less than a conservative threshold 
$s_{\max }$ of $5 \%$ of the water depth. Tension in the lines must stay positive. Accumulated fatigue damage at the top of each line must remain below a resistance $R$.

For a fixed sea state $\left(h_{s}, t_{p}, u\right)_{i}$, the movements of the platform and the lines are solutions of a linearized movement equation in which the forces come from environmental loads. The surge and tension in each line can be seen as outputs of linear filters with the sea elevation $\eta_{\left(h_{s}, t_{p}, u\right)_{i}}$ as input. The surge $S_{i}$ and tension $\mathcal{T}_{i}$ are the convolution of a response function (respectively $h_{\mathcal{S}}$ and $h_{\mathcal{T}}$ ) and $\eta_{\left(h_{s}, t_{p}, u\right)_{i}}$ :

$$
\mathcal{S}_{i}(t)=h_{\mathcal{S}} * \eta_{\left(h_{s}, t_{p}, u\right)_{i}}(t), \quad \mathcal{T}_{i}(t)=h_{\mathcal{T}} * \eta_{\left(h_{s}, t_{p}, u\right)_{i}}(t) .
$$

Taking into account the different sea states, the surge and the tension on $[0, T]$, denoted $\mathcal{S}$ and $\mathcal{T}$, are defined as the sum of solutions of Equation 1 on each interval $I_{i}$ as follows:

$$
S(t)=\sum_{i=1}^{n_{T}} S_{i}(t) 1_{I_{i}}(t), \quad \mathcal{T}(t)=\sum_{i=1}^{n_{T}} \tau_{i}(t) 1_{I_{i}}(t)
$$

Furthermore, the accumulated damage $\mathcal{F}_{[0, T]}$ in the line during the time interval $[0, T]$ can be estimated from the variation of the tension process. It is a positive scalar and failure is considered when it exceeds $R$. We will see in section 3.3 how this quantity can be directly estimated from the first spectral moments of the tension, following a frequency domain simulation.

To simplify the notations, in the presentation of the problem and of our methodology, we consider only the tension and the fatigue at the top of one mooring line since the methodology is similar for each line.
However, in the resolution we consider them for each of the three mooring lines.
To account for the lack of knowlddge on certain parameters, uncertainties are eonsidered on:

- the wave azimuth which is represented by a random variable $X_{p_{1}}$ uniformly distributed between

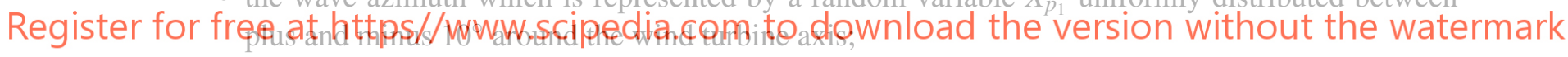

- two quadratic damping coefficients for the surge and the pitch of the floater, denoted $X_{p_{2}}$ and $X_{p_{3}}$, to account for the approximation of the fitting method from decay tests [7]. Each of these random variables follows a uniform law respectively on $\left[10^{5}, 10^{6}\right]\left(\right.$ in $\left.\mathrm{N} . \mathrm{s}^{2} . \mathrm{m}^{-2}\right)$ and $\left[3 \times 10^{10}, 7 \times 10^{10}\right]$ (in N.m.s ${ }^{2} \cdot \mathrm{rad}^{-2}$ );

- the $y$-intercept of the fatigue law $X_{d_{2}}$ accounting for experimental scattering. It follows a lognormal distribution with parameters $\sigma_{d_{2}}=0.8$ and $\mu_{d_{2}}$ which depends on the lineic mass $d_{2}$ according to a relation described in [8] ;

- the threshold resistance $R$ for approximation of time independent Palmer Miner damage approach. It is a log-normal distribution of parameters $\mu_{R}=1$ and $\sigma_{R}=0.3$ [9].

All of these variables are independent and $X_{p}$ denotes the random vector such that $X_{p}=\left(X_{p_{1}}, X_{p_{2}}, X_{p_{3}}\right)$. 


\subsection{Optimization problem formulation}

Taking into account all the sources of uncertainty, we decided to consider probabilistic constraint and we end up with the RBDO problem (3) under study in this paper.

$$
\begin{aligned}
& \min _{d \in \Omega_{d}} \operatorname{cost}(d) \quad \text { such that } \\
& \mathbb{P}\left(\max _{[0, T]} \mathcal{S}\left(d, X_{p}, \eta_{X_{L T}}, t\right)>s_{\max }\right)<10^{-4} \\
& \mathbb{P}\left(\min _{[0, T]} \mathcal{T}\left(d, X_{p}, \eta_{X_{L T}}, t\right)<0\right)<10^{-4} \\
& \left.\mathbb{P}\left(\mathcal{F}_{[0, T]}\left(d, X_{p}, X_{d_{2}}, \eta_{X_{L T}}\right)\right)>R\right)<10^{-4}
\end{aligned}
$$

The main difficulty to solve this problem is to evaluate at each iteration of the optimization algorithm the low failure probabilities which require many simulations, each of them giving a realization of $\mathcal{S}, \mathcal{T}$ and $\mathcal{F}_{[0, T]}$.

\section{CONSTRAINTS REFORMULATION}

We propose in this paper a new formulation based on Extreme Value Theory, which is not to our knowledge proposed in the RBDO methods of the literature.

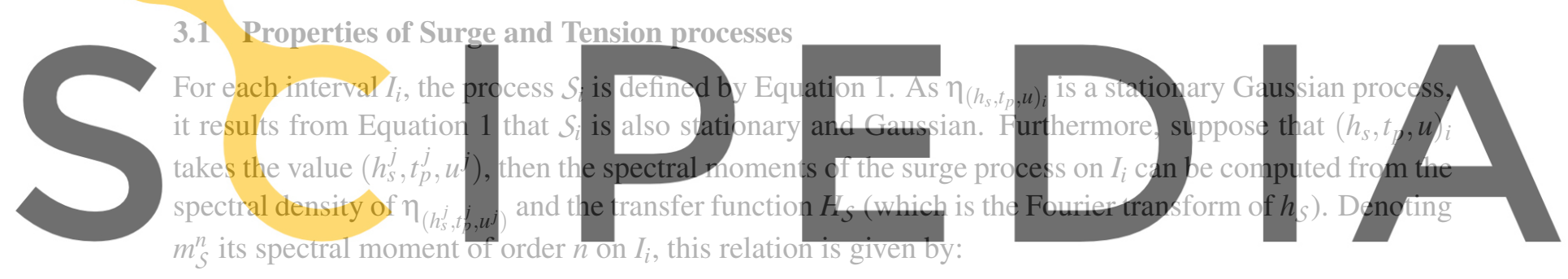

Register for free at https//www.scipedia.com to download the version without the watermark $m_{S}^{n}\left(h_{s}^{j}, t_{p}^{\prime}, u^{\top}\right)=\int_{\mathbb{R}} \omega^{n}\left|H_{S}(\omega)\right|^{2} \psi_{j}(\omega) d \omega$.

In Equation 4, $\psi_{j}$ is the spectral density of $\eta_{\left(h_{s}^{j}, t_{p}^{j}, u^{j}\right)}$. The standard deviation of the surge process is the square root of its 0 -th spectral moment and will be denoted $\sigma_{\mathcal{S}}$.

For the same reasons, $\mathcal{T}_{i}$ is also a stationary Gaussian process and its spectral moments are computable from the spectral density of $\eta_{\left(h_{s}^{j}, t_{p}^{j}, u^{j}\right)}$ and the transfer function $H_{\mathcal{T}}$.

The mean of the surge and tension processes, denoted respectively $\mu_{\mathcal{S}}$ and $\mu_{\mathcal{T}}$, are computed by the static simulation (see [4] for more details).

These properties will be useful for the reformulation of each constraint.

\subsection{Constraints of extreme value type}

An important theorem from the Extreme Value Theory states that for a standardized stationary Gaussian process $\zeta$ and under certain conditions on its spectral density detailed in [10], there exist two deterministic functions $a_{T}$ and $b_{T}$ which depend on $T$ and the second spectral moment of $\zeta$ such that 


$$
\mathbb{P}\left(a_{T}\left(\max _{[0, T]} \zeta(t)-b_{T}\right) \leq \alpha\right) \rightarrow \exp \left(-\exp ^{-\alpha}\right) \text { as } T \rightarrow \infty
$$

Furthermore, we have seen in the section 3.1 that the process $\mathcal{S}_{i}$ is stationary and Gaussian on an interval of length $\Delta T$. Then, by standardizing $\mathcal{S}_{i}$ of mean and standard deviation $\mu_{\mathcal{S}}$ and $\sigma_{\mathcal{S}}$, we can apply (5) to obtain

$$
\mathbb{P}\left(\max _{[0, \Delta T]} S_{i}(t)>S_{\max }\right) \simeq F\left(e^{a_{\Delta T}\left(b_{\Delta T}-\frac{s_{\max }-\mu_{\mathcal{S}}}{\sigma_{\mathcal{S}}}\right)}\right)
$$

with $F(x)=1-\exp (-x)$.

Using (6) and with calculations that we will not detail in this paper, for a fixed $x_{p}$, the probability that $\mathcal{S}$ exceeds $s_{\max }$ over the period $[0, T]$ can be approximated by

$$
F\left(\sum_{j=1}^{7} \exp \left(a_{T p^{j}}\left(b_{T p^{j}}\left(d, x_{p}, h_{s}^{j}, t_{p}^{j}, u^{j}\right)-\frac{s_{\max }-\mu_{\mathcal{S}}\left(d, x_{p}, h_{s}^{j}, t_{p}^{j}, u^{j}\right)}{\sigma_{S}\left(d, x_{p}, h_{s}^{j}, t_{p}^{j}, u^{j}\right)}\right)\right)\right)
$$

where $p^{j}$ is the probability of occurrence of the $j$-th sea state $\left(h_{s}^{j}, t_{p}^{j}, u^{j}\right)$.

The same reasoning is applied to reformulate the tension constraint.

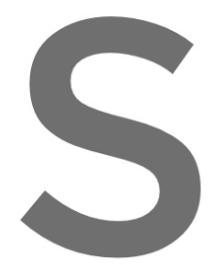

3.3 Fatigue estimation with the Dirlik method

As we use frequency domain simulations, the contr

with the usual RainFlow-Miner's

formula estimates the distribution of tension cycle
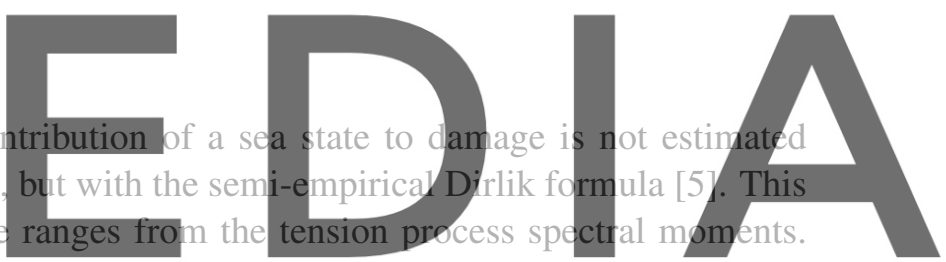

For a design $d$ and fixed uncertainties $x_{p}$ and $x_{d_{2}}$, only the four first spectral moments of the process are

Register for free at https//WWW.Scipedia.com to downloaid the version without the watermark

$$
\mathbb{E}\left[\mathcal{F}_{[0, T]}\left(d, x_{p}, \eta_{h_{s}^{j}, t_{p}^{j}, u^{j}}\right)\right] \simeq \frac{\mathcal{D}_{[0, T]}\left(d, x_{p}, h_{s}^{j}, t_{p}^{j}, u^{j}\right)}{x_{d_{2}}} .
$$

The expression of $\mathcal{D}_{[0, T]}$ is analytically given from the spectral moments of the tension process [11].

\subsection{Problem reformulation}

Using the Extreme Value Theory for the two first constraints and the Dirlik approach for the fatigue we obtain a new formulation of the constraints given in (9) equivalent to the initial constraints in (3). 


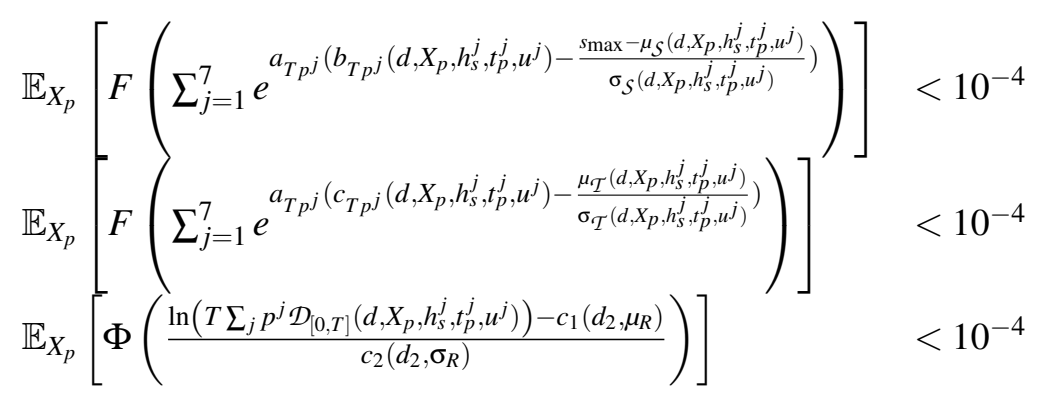

Here, we recall that $a_{T p^{j}}, b_{T p^{j}}, c_{T p^{j}}$ are functions that only depend on $T, p^{j}$ and the second spectral moments of the surge and tension process for the $j$-th sea state. The evaluation of the means and standard deviations $\mu_{\mathcal{S}}, \mu_{\mathcal{T}}, \sigma_{\mathcal{S}}, \sigma_{\mathcal{T}}$ is described in section 3.1. The calculation of $\mathcal{D}_{[0, T]}$ only requires the spectral moments of the tension process. And finally $c_{1}$ and $c_{2}$ are constants depending on $d_{2}, \mu_{R}$ and $\sigma_{R}$ that result from the integration of the uncertainties on $X_{d_{2}}$ and $R$.

We point out that, for a design $d$, a fixed variable $x_{p}$, we only need the spectral moments of the surge and tension process for each sea state to evaluate the quantities into square brackets in (9). These statistical properties depend on the spectral density of the sea elevation (which is known) and the transfer functions $H_{\mathcal{S}}$ and $H_{\mathcal{T}}$ which only require one call to the expensive simulator to be obtained (for each $d$ and $x_{p}$ ). It is much more effective to solve the problem with the new formulation since to evaluate the quantities into brackets of the initial problem (3), we need to sample many realizations (and call as many times the

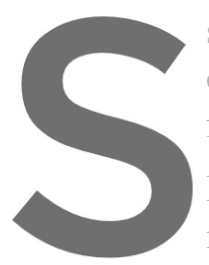
simulator) of the surge ancenten only depend on the uncerti from time-dependent cons However, it is still time method. Over the last decar
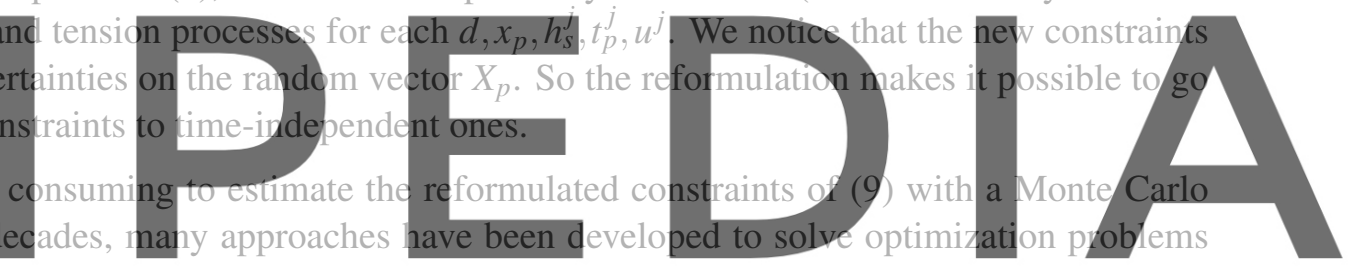

with failure constraints among which methods based on an adaptive kriging (AK) strategy $[12,13,14]$.

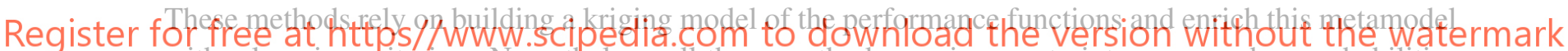
with a learning criterion. Nevertheless, all these methods require constraints expressed as probabilities while the constraints are expressed as expectations in our reformulation approach, as seen in (9). This particularity has motivated the implementation of a new AK method called AK-ECO for "Adaptive Kriging method for Expectation Constraint Optimization" that we introduce in the next section.

\section{RESOLUTION OF THE REFORMULATED PROBLEM}

Given that evaluating the reformulated constraints expressed in (9) with Monte Carlo is still time consuming, a metamodel is built to replace the expensive functions of each constraint. The AK-ECO procedure consists in carrying out cycles of optimization in which a first phase of local enrichment of the metamodels is performed followed by a resolution of the reformulated problem using Monte Carlo with the refined metamodels. 


\subsection{Metamodel strategy}

Let us consider the first reformulated constraint. We define the function $M_{\mathcal{S}}$ such that:

$$
M_{\mathcal{S}}\left(d, x_{p}, h_{s}^{j}, t_{p}^{j}, u^{j}\right)=a_{T p^{j}}\left(b_{T p^{j}}\left(d, x_{p}, h_{s}^{j}, t_{p}^{j}, u^{j}\right)-\frac{s_{\max }-\mu_{\mathcal{S}}\left(d, x_{p}, h_{s}^{j}, t_{p}^{j}, u^{j}\right)}{\sigma_{\mathcal{S}}\left(d, x_{p}, h_{s}^{j}, t_{p}^{j}, u^{j}\right)}\right) .
$$

Since one evaluation of $M_{\mathcal{S}}$ requires a call to the simulator, we can replace $M_{\mathcal{S}}$ by a metamodel to speed up the evaluation of the first constraint. Kriging models have been intensively used in reliability analysis because they not only provide a prediction but also an information about the quality of this prediction. In fact, a kriging model of $M_{\mathcal{S}}$ is expressed at a point $\left(d, x_{p}, h_{s}^{j}, t_{p}^{j}, u^{j}\right)$ as a normal random variable representing the uncertainties of prediction of $M_{\mathcal{S}}$. Its mean is used as predictor and its standard deviation as a indicator of the precision of the metamodel at this point.

The calibration of the kriging model of $M_{S}$ is carried out on the space $\Omega_{d} \times \Omega_{P} \times \Omega_{L T}$ of dimension 9 where $\Omega_{P}$ and $\Omega_{L T}$ respectively refer to the sample spaces of $X_{p}$ and $\left(H_{S}, T_{p}, U\right)$. Furthermore, the triplet $\left(H_{S}, T_{p}, U\right)$ have 7 possible outcomes that are well ordered and this order is conserved when we replace the triplet outcomes by the corresponding outcome of $H_{S}$. Doing so, we reduced the dimension of $\Omega_{L T}$ from 3 to 1 . Thus the metamodel is actually built in a 7-dimensional space.

For the second and third constraints, the metamodels will apply to the functions $M_{\mathcal{T}}$ and $M_{\mathcal{D}}$ defined by Equations 11-12.
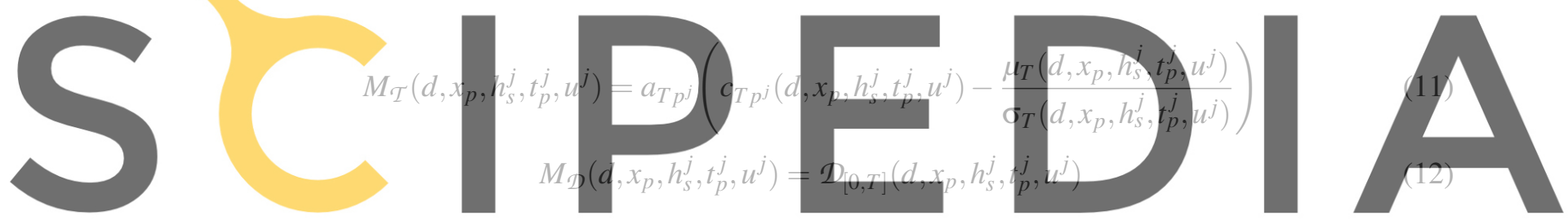

We notice that the three metamodels are carried out on the same space and that one call to the simulator

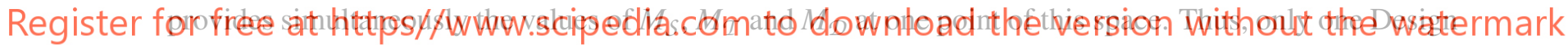
of Experiments (DoE) is enough to calibrate the three metamodels and will be shared at each enrichment step.

\subsection{AK-ECO procedure}

\subsubsection{Initialization}

The procedure begins with the choice of an initial design point $d^{0}$ from which the optimization will start. Moreover initial krigings must be built for $M_{\mathcal{S}}, M_{\mathcal{T}}$ and $M_{\mathcal{D}}$ which require the computation of a spacefilling DoE denoted $D o E^{0}$. Once $D o E^{0}$ is obtained, the kriging models ${\widetilde{M_{\mathcal{S}}}}^{0},{\widetilde{M_{\mathcal{T}}}}^{0},{\widetilde{M_{\mathcal{D}}}}^{0}$ are calibrated and the first cycle of optimization $(k=1)$ can begin.

\subsubsection{Sequential cycles of optimization}

Once the initialization is complete, the reformulated problem is solved through sequential cycles of optimization. Each cycle is numbered $k$ and is decomposed into two steps. We respectively denote $d^{k-1}$, 
$D o E^{k-1},{\widetilde{M_{\mathcal{S}}}}^{k-1},{\widetilde{M_{\mathcal{T}}}}^{k-1},{\widetilde{M_{\mathcal{D}}}}^{k-1}$ the design point, DoE and kriging models recovered from the initialization for $k=1$ or from the previous cycle for $k>1$.

Step 1. Local enrichment at $d^{k-1}$ of the metamodels ${\widetilde{M_{\mathcal{S}}}}^{k-1},{\widetilde{M_{\mathcal{T}}}}^{k-1},{\widetilde{M_{\mathcal{D}}}}^{k-1}$ :

- Step 1.a. An accuracy criterion assesses the precision of each metamodel at $d^{k-1}$ (we detail this step in section 4.2.3)

- Step 1.b. For each inaccurate metamodel, one local enrichment is carried out. The local refinement of each metamodel consists in adding to the shared DoE the point $\left(x_{p}, h_{s}^{j}, t_{p}^{j}, u^{j}\right)$ selected by the procedure described in section 4.2.4. The simulator is evaluated at the selected point. This point and its response are added to the previous DoE to obtain a new DoE from which all the kriging models are recalibrated.

Steps 1.a and 1.b are repeated until each kriging model meets the accuracy condition of step 1.a. At the end of step 1, the enriched DoE and kriging models are denoted $D o E^{k}, \widetilde{M}_{\mathcal{S}}{ }^{k},{\widetilde{M_{\mathcal{T}}}}^{k}$ and ${\widetilde{M_{\mathcal{D}}}}^{k}$.

Step 2. The reformulated problem is solved using the optimization algorithm chosen by the user starting from $d^{k-1}$. At each iteration of the optimization, the constraints are estimated through Monte Carlo on the current surrogates ${\widetilde{M_{\mathcal{S}}}}^{k},{\widetilde{M_{\mathcal{T}}}}^{k}$ and ${\widetilde{M_{\mathcal{D}}}}^{k}$. This step does not require any call to the expensive simulator. Once the optimization algorithm has converged, a new design denoted $d^{k}$ is provided.

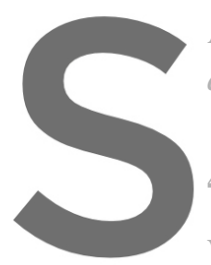

At the end of each cycle $k$, $d^{\min }$ is $d^{k}$, otherwise, $k$

4.2.3 Details of step

We introduce in this section the accuracy conditi

$k$-th cycle of optimization, a kriging model $\widetilde{M}_{S}^{k-1}$ is recovered from the previous cycle. The prediction

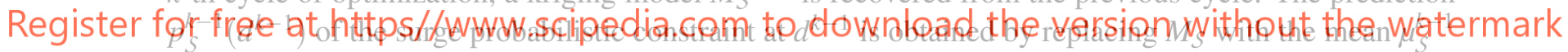

of $\widetilde{M}_{S}^{k-1}$. As in Dubourg (2011), the standard deviation $\sigma_{S}^{k-1}$ of $\widetilde{M}_{S}^{k-1}$ can be used to obtain a low, resp. high, estimator $\underline{p}_{S}^{k-1}\left(d^{k-1}\right)$, resp. $\bar{p}_{\mathcal{S}}^{k-1}\left(d^{k-1}\right)$, of the surge constraint at $d^{k}-1$ by replacing $M_{\mathcal{S}}$ with $\mu_{\mathcal{S}}^{k-1}+2 \sigma_{\mathcal{S}}^{k-1}$ or with $\mu_{\mathcal{S}}^{k-1}-2 \sigma_{\mathcal{S}}^{k-1}$. The kriging model ${\widetilde{M_{\mathcal{S}}}}^{k-1}$ is considered accurate at $d^{k-1}$ if the following condition is met.

$$
\frac{\left|p_{\mathcal{S}}^{k-1}\left(d^{k-1}\right)-10^{-4}\right|}{\left|\bar{p}_{\mathcal{S}}^{k-1}\left(d^{k-1}\right)-\underline{p}_{\mathcal{S}}^{k-1}\left(d^{k-1}\right)\right|}>1
$$

Therefore the kriging model is considered sufficiently accurate if the distance between the low and high estimations of the constraint is less than the distance between the estimation of the constraint and $10^{-4}$. When this condition is met, we have reasonable grounds to believe that the kriging model accurately predicts whether a point near $d^{k-1}$ belongs or not to the safe space.

The criteria for the others constraints are adapted for each constraint formulation following the same reasoning. 


\subsubsection{Details of step 1.b}

During the $k$-th cycle, if $\widetilde{M}_{\mathcal{S}}^{k-1}$ is not accurate at $d^{k-1}$, a point of the space $\Omega_{P} \times \Omega_{L T}$ is selected by maximizing a criterion $C_{S}^{k}$ :

$$
\left(x_{p_{e n r}}, h_{s}^{e n r}, t_{p}^{e n r}, u^{e n r}\right)=\arg \max _{\Omega_{p} \times \Omega_{L T}} C_{\mathcal{S}}^{k}\left(x_{p}, h_{s}, t_{p}, u\right) .
$$

The criterion $C_{S}^{k}$ favors points where the uncertainty of prediction implies important uncertainties on the constraint estimation at $d^{k-1}$. The simulator is then called at $\left(d^{k-1}, x_{p_{e n r}}, h_{s}^{e n r}, t_{p}^{e n r}, u^{e n r}\right)$ and this point and the response at this point are added the current DoE.

\subsection{Implementation of AK-ECO}

To solve the reformulated problem, the procedure described in section 4.2 has been implemented using a Latin Hypercube Sampling [15] of size 70 for the initial DoE. A maximum of 15 local enrichments has been imposed at each cycle and for each constraint which stops the enrichment of the concerned constraint even if the criterion of accuracy is not satisfied. Moreover each constraint is refined at least once at each cycle even if the criterion of accuracy is met. The optimization algorithm used in step 2 is the COBYLA (Constrained Optimization BY Linear Approximations) algorithm [16]. The constraints are estimated with a Monte Carlo sample of size 30,000. The AK-ECO procedure is finished if $\| d^{k-1}-$ $d^{k} \|<10^{-2}$.
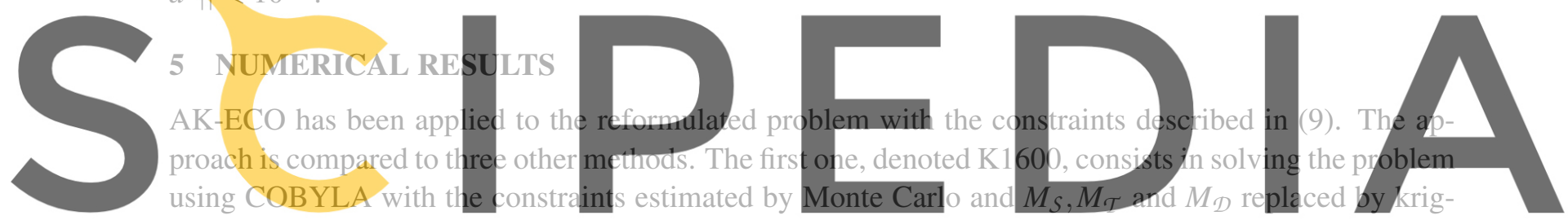

ing models built from a DoE of size 1600. No refinement is done in this method which will provide a

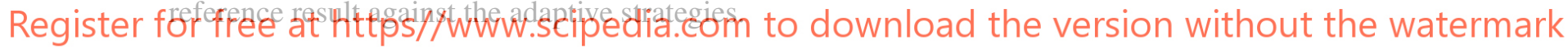

The problem is also solved with the SORA method [17] and a method proposed by Stieng in [18]. They

both require a formulation of the constraints as probabilities which can be obtained by noticing that the three reformulated constraints are written as $\mathbb{E}_{X_{P}}\left[F_{c}\left(g\left(d, X_{p}\right)\right)\right]$ with $F_{c}$ a cumulative distribution function (exponential for the first constraints and normal for the last one). They are also based on the resolution of a RBDO problem through cycles of optimization. In SORA, the reliability constraints are converted into deterministic ones by fixing the uncertainties to certain values during the optimization. At the end of each cycle, these values are updated and a new cycle of optimization begins at the design point obtained at the previous cycle. The second method relies on an approximation of the performance function by the product of two functions: one depending only on the design variables and the other one depending only on the uncertain variables. A metamodel is fitted on the second function and is updated at each cycle of optimization.

All the methods have been initialized at the same design point $d^{0}=(-1.07,138.9,0.07)$. The AK-ECO and Stieng algorithms were terminated because the changes in design between two consecutive cycles were small enough. For SORA, the limit of two days of calculation was reached, which stopped the algorithm. The results obtained are presented in Table 1 . The quantities $p_{\mathcal{S}}\left(d^{\text {min }}\right)$ and $p_{\mathcal{F}}^{\text {line }}\left(d^{\text {min }}\right)$ are the 
failure probabilities for surge and damage at top of mooring line 3, respectively. They are estimated at $d_{\min }$ for each method using a massive Monte Carlo with the kriging models built for the K1600 method. In Table 1 , all the other constraints are not represented as they are equal to zero at $d_{\min }$ i.e. they are not active for the chosen case study. The number of calls to the expensive simulator is denoted by $N_{\text {calls }}$.

Table 1: Numerical results

\begin{tabular}{|c|c|c|c|c|}
\hline & K1600 & SORA & Stieng & AK-ECO \\
\hline$d^{\text {min }}$ & $(1.11,110.45,0)$ & $(-0.2,101.2,0)$ & $(-1.99,86.6,0)$ & $(1.05,109.96,0)$ \\
\hline cost $\left(d^{\text {min }}\right)$ & 0.262 & 0.201 & 0.104 & 0.259 \\
\hline$p_{\mathcal{S}}\left(d^{\text {min }}\right)$ & $1.0 \times 10^{-4}$ & $0.9 \times 10^{-4}$ & 0.66 & $1.0 \times 10^{-4}$ \\
\hline$p_{\mathcal{F}}^{\text {line }_{3}}\left(d^{\text {min }}\right)$ & $1.0 \times 10^{-4}$ & $2.6 \times 10^{-4}$ & $14.2 \times 10^{-4}$ & $1.0 \times 10^{-4}$ \\
\hline$N_{\text {calls }}$ & 1600 & 24983 & 4557 & 456 \\
\hline
\end{tabular}

The results presented in Table 1 show that AK-ECO requires much less calls to the simulator than the other approaches to provide a a design configuration that is both optimized and reliable: 70 calls are needed for the initial DoE and 386 for the enrichments of the metamodels. Moreover the estimation of the constraints is more accurate with AK-ECO. The lack of precision of the two competing methods can be explained by the reliability method used in SORA to update the uncertainties and in Stieng to estimate the constraints: algorithm leading to impo for $p s$ since this constraint impose a maximum of 10 models are used for the for the second, 100 for the
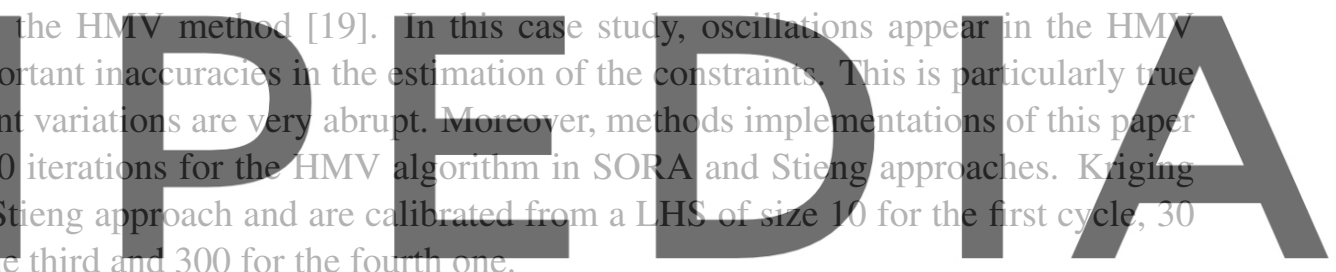

Register for free at https//www.scipedia.com to download the version without the watermark

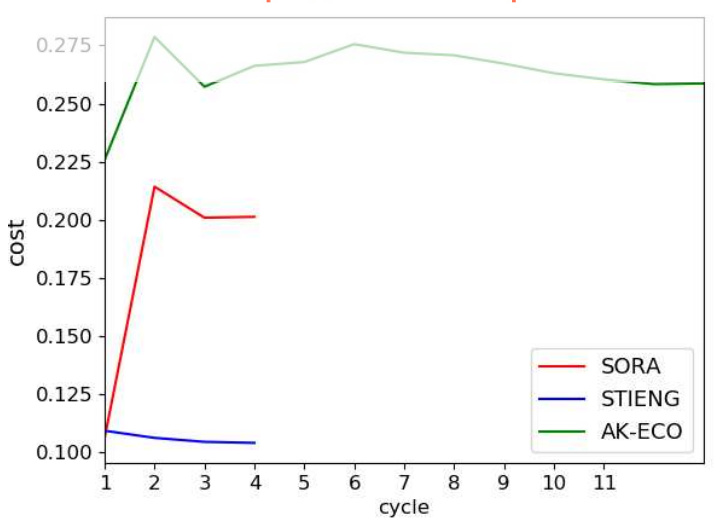

(a) Normalized cost function

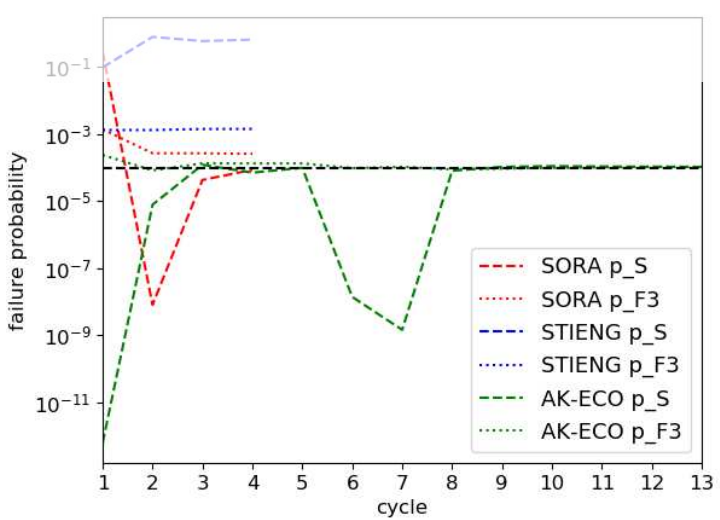

(b) Failure probabilities

Figure 1: Cost function and failure probabilities at the end of each cycle 
We display in Figure 1 the evolution of the normalized cost function (Figure 1a) and the active constraints $p_{\mathcal{S}}$ and $p_{\mathcal{F}}^{\text {line }}{ }^{3}$ (Figure $1 \mathrm{~b}$ ) at the design points obtained at the end of each cycle for each algorithm. The probabilities are still estimated using Monte Calo with the kriging models calibrated for the K1600 method. We observe that at each cycle, our implementations of SORA and Stieng methods obtain lower values of the cost function but this is due to poor estimations of the constraints. On the other hand, the design obtained by AK-ECO at the end of each cycle always respect the real constraints: the failure probabilities stay under $10^{-4}$.

We emphasize that the conclusions of the comparison presented in this article apply to the particular case presented here and the chosen configurations of the different algorithms. More work need to be done to extend our remarks to other applications and to investigate the dependency on the initial state with a multi-start strategy.

\section{CONCLUSION}

This paper presents a new two-step methodology to minimize the cost of the mooring system of a FOWT under probabilistic constraints. First, the linearity of the movement equation allows to transmit the piecewise stationarity of the sea elevation process to the surge and tension processes. The Extreme Value Theory is then used to reformulate the constraints involving the extrema of these processes. Furthermore, the Dirlik approach is implemented to evaluate the fatigue constraint. At the end of this reformulation step, new constraints are expressed as expectations. The existing methods in reliability analysis are based on the fact that the constraints are written as probabilities and hence are not suited for our problem. Therefore a new adaptive method is proposed in this paper to estimate efficiently the reformulated constraints.

This methodology is applied with success to a representative Floating Wind Turbine case study. The reformulated problem is solved with AK-ECO and three others approaches in section 4. Compared to the alternatives, the results show that AK-ECO is best suited to solve the reformulated problem.

\section{REFERENCES}

[1] Robertson, A., Jonkman, J., Masciola, M., Song, H., Goupee, A., Coulling A. and Luan, C. Definition of the Semisubmersible Floating System for Phase II of OC4 (No. NREL Technical report NREL/TP-5000-60601) (2014).

[2] Det Norske Veritas. Design of floating wind turbine structures. Offshore Standard DNV-OS-J103 (2013).

[3] Vorpahl, F., Schwarze, H., Fischer, T., Seidel, M., and Jonkman, J. Offshore wind turbine environment, loads, simulation, and design. Wiley Interdisciplinary Reviews: Energy and Environment (2013), 2(5):548-570.

[4] Le Cunff, C., Ryu, S., Heurtier, J. M. and Duggal, A. S. Frequency-Domain Calculations of Moored Vessel Motion Including Low Frequency Effect. International Conference on Offshore Mechanics and Arctic Engineering (2008), 48180:689-696.

[5] Dirlik, T. Application of computers in fatigue analysis. PhD thesis, University of Warwick (1985).

[6] Hasselmann, D.E., Dunckel, M. and Ewing, J.A. Directional wave spectra observed during JON- 
SWAP 1973. Journal of physical oceanography (1980), 10(8):1264-1280.

[7] Burmester, S., Vaz, G., Gueydon, S. and el Moctar, O. Investigation of a semi-submersible floating wind turbine in surge decay using CFD. Ship Technology Research (2020), 67(1):2-14.

[8] Rossi, R. R. A review of fatigue curves for mooring lines. ASME 2005 24th International Conference on Offshore Mechanics and Arctic Engineering (2005) 1097-1104.

[9] Leira, B. J., Igland, R. T., Baarholm, G. S., Farnes, K. A. and Percy, D. Fatigue safety factors for flexible risers based on case specific reliability analysis. ASME 2005 24th International Conference on Offshore Mechanics and Arctic Engineering (2005) 211-217.

[10] Leadbetter, M.R., Lindgren, G. and Rootzén, H. Extremes and Related Properties of Random Sequences and Processes. Springer series in statistics (1983).

[11] Benasciutti, D. and Tovo, R. Rainflow cycle distribution and fatigue damage in Gaussian random loadings. Internal Report No. 129, Department of Engineering, University of Ferrara, Italy (2004).

[12] Dubourg, V. Adaptive surrogate models for reliability analysis and reliability-based design optimization. PhD thesis, Université Blaise Pascal - Clermont-Ferrand, France (2011).

[13] Moustapha, M. and Sudret, B. Surrogate-assisted reliability-based design optimization: a survey and a unified modular framework. Structural and Multidisciplinary Optimization (2019), 60(5):2157--2176.

[14] Lelièvre, N., Beaurepaire, P., Mattrand, C. and Gayton, N. AK-MCSi: A Kriging-based method to deal with small failure probabilities and time-consuming models. Structural Safety (2018), 73:1-11.

[15] McKay, M. D., Beckman, R. J. and Conover, W. J. A Comparison of Three Methods for Selecting Values of Input Variables in the Analysis of Output from a Computer Code. Technometrics (1979), 21(2): 239-245.

[16] Powell, Michael JD. A direct search optimization method that models the objective and constraint functions by linear interpolation. Advances in optimization and numerical analysis. Springer, Dordrecht (1994). 51-67.

[17] Du, X. and Chen, W. Sequential optimization and reliability assessment method for efficient probabilistic design. Journal of Mechanical Design (2004), 126(2):225-233.

[18] Stieng, L.E.S. Optimal design of offshore wind turbine support structures under uncertainty. $\mathrm{PhD}$ thesis, Norwegian University of Science and Technology (2019).

[19] Youn, B.D., Choi, K.K and Park, Y.H. Hybrid analysis method for reliability-based design optimization. Journal of Mechanical Design (2003), 125(2):221-232.

\section{ACKNOWLEDGMENTS}

The authors would like to thank J.M. Heurtier from Principia for his help in the post-processing of the computation in frequency domain of DeeplinesWind ${ }^{\mathrm{TM}}$, Y. Poirette from IFPEN for his help in the definition in the optimization problem and the adaptation of the DeeplinesWind ${ }^{\mathrm{TM}}$ model and P. Bozonnet from IFPEN for her help in the definition of the hydrodynamic damping coefficient uncertainties. 